\title{
Biofeedback Therapy in Constipation and Fecal Incontinence
}

\author{
Seung-Jae Myung, MD, PhD \\ Division of Gastroenterology, Constipation Clinic, Asan Digestive Disease Research Institute, Asan Medical Center, University of Ulsan College \\ of Medicine, Seoul, Korea
}

Article: The long-term clinical efficacy of biofeedback therapy for patients with constipation or fecal incontinence (J Neurogastroenterol Motil 2010;16:177-185)

Chronic constipation and incontinence are common symptoms especially in western countries. ${ }^{1}$ However, these problems are increasing among Asians probably due to the rise in aging population and westernization of diet. The pathogenesis of constipation varies in patients with colonic transit delay, dyssynergic defecation, or both, while the cause of incontinence is often weakening or structural defect of anal sphincters. ${ }^{2}$

Constipated patients may respond either to fiber-fluid supplementation, prokinetics, or judicious use of laxatives. Among non-responders, outlet obstructive type of dysfunction seems particularly common; it affects up to $50 \%$ of patients with chronic constipation who are referred to a tertiary care center. ${ }^{3}$ In patients with incontinence, biofeedback therapy is the first line treatment before considering surgery. ${ }^{3}$

Biofeedback therapy is a learning strategy originating from psychological learning theories, more specifically from instrumental learning' and 'operant conditioning." ${ }^{4}$ This therapy has been reported to be effective in constipation and incontinence. Recently, three randomized controlled trials (RCTs) have been published between 2006 to 2007 which provide definitive evi- dence for the efficacy of biofeedback in adults with dyssynergic defecation. ${ }^{5-7}$ Biofeedback treatment is also effective in reducing incontinence in more than two-thirds of patients and is generally well accepted. ${ }^{2}$

In this issue of Journal of Neurogastroenterology and Motility, Lee et al. reported the result of biofeedback therapy for patients with constipation and incontinence in a single center. Their report indicated unsatisfactory symptom improvements for both constipation and incontinence, although the improvement lasted at least a year in patients with excellent responses. This is rather a disappointing news for patients with constipation and incontinence waiting for hopeful results of novel treatment. However, it is too early to be disappointed.

When we read an article regarding the results of treatment in specific patients group, several factors such as enrolled patients group (strict diagnostic criteria), treatment method, and evaluation of treatment efficacy should be carefully considered. I suggest to meticulously analyze these factors in this article before considering biofeedback treatment is as not so effective for constipation and incontinence.

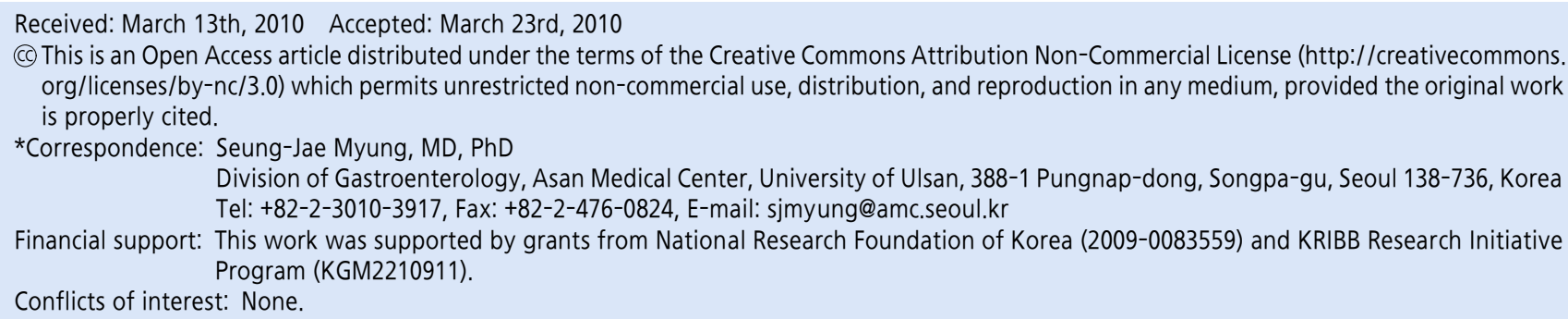


Firstly, not every enrolled patient might have been exactly suitable for biofeedback therapy. In randomized controlled trial, this therapy is effective in 70 to $86 \%$ of patients with dyssynergic defecation. ${ }^{5-7}$ The diagnostic criteria for dyssynergic defecation is well described in a recent review, ${ }^{8}$ which includes dyssynergic anorectal manometric findings reflecting abnormal relexation pattern or inadequate propulsive force plus abnormal findings from balloon expulsion test, defecography, or colon transit time. ${ }^{8}$ It is proven in RCT that biofeedback benefits only patients with outlet dysfunction, and no patients with isolated slow transit constipation. ${ }^{9}$ In this article, as the authors mentioned in the discussion, the diagnostic criteria for constipation were not defined, which could have led to the low response rate of biofeedback among the enrolled constipated patients since patients with slow transit constipation might have been included. It is understandable to try biofeedback therapy in medically intractable constipation. However, analyzing the response rate of the therapy according to the pathogenesis of the diseases would be beneficial. Our group also tried to evaluate the variables to predict biofeedback responsiveness and found the defecation index and pelvic floor dyssynergia to be the factors influencing the responses by multivariate analysis. ${ }^{10}$ However, there is no consensus for markers in predicting the biofeedback response. In the future, we should set up protocols to select the suitable patients for biofeedback therapy using adequate biomarkers.

Secondly, the method and protocol of biofeedback therapy should be reviewed. The effectiveness of biofeedback training depends in part on the skills of the biofeedback therapist and the selection of particular techniques used for the training. ${ }^{2}$ In a recent RCT, the patients were treated by a physician who highly experienced in biofeedback training. ${ }^{11}$ The description of the biofeedback protocol was adequate in this article although there is a possibility that better protocol and method to induce more fair response. In addition, this protocol lacked the sessions for sensory retraining or strengthening of propulsive force for indicated patients. $^{12}$

Thirdly, the evaluation criteria for the response rate are important. The subjective reporting system including 4 categories used by authors are reasonable and widely used. However, the evaluation of efficacy including objective and subjective informations will add to the knowledge on the effectiveness of this therapy.

They reported intriguing observation of a higher sensory volume in nonresponsive than in responsive group. As the authors have discussed, there might be a possibility that rectal hypo- sensitivity is one of the parameters to predict poor response of biofeedback therapy. ${ }^{12}$ Our group suggested electrical stimulation to be helpful for patients with rectal hyposensitivity, especially among those who were unresponsive to biofeedback therapy. ${ }^{13,14}$ Further study is warranted to evaluate the importance of this novel mechanism in constipation.

In summary, the article by Lee et al. provided valuable information about biofeedback therapy in Korean patients with constipation and incontinence. However, the overall response rate might have been underestimated due to patient's selection and/or short treatment period. The promising finding is the long-term maintenance of improvement in the responsive patients. They also suggested rectal hyposensitivity as a possible biomarker for predicting biofeedback responsiveness which is a valuable and plausible hypothesis. Further study is warranted to evaluate the effectiveness of biofeedback therapy and to develop biomarkers to predict the response of this therapy in Asian patients with constipation and incontinence.

\section{References}

1. Lembo A, Camilleri M. Chronic constipation. N Engl J Med 2003;349:1360-1368.

2. Enck P, Van der Voort IR, Klosterhalfen S. Biofeedback therapy in fecal incontinence and constipation. Neurogastroenterol Motil 2009;21:1133-1141.

3. Chiarioni G, Whitehead WE. The role of biofeedback in the treatment of gastrointestinal disorders. Nat Clin Pract Gastroenterol Hepatol 2008;5:371-382.

4. Glia A, Gylin M, Gullberg K, Lindberg G. Biofeedback retraining in patients with functional constipation and paradoxical puborectalis contraction: comparison of anal manometry and sphincter electromyography for feedback. Dis Colon Rectum 1997;40:889-895.

5. Rao SS, Seaton K, Miller M, et al. Randomized controlled trial of biofeedback, sham feedback, and standard therapy for dyssynergic defecation. Clin Gastroenterol Hepatol 2007;5:331-338.

6. Heymen S, Scarlett Y, Jones K, Ringel Y, Drossman D, Whitehead WE. Randomized, controlled trial shows biofeedback to be superior to alternative treatments for patients with pelvic floor dyssynergia-type constipation. Dis Colon Rectum 2007;50:428-441.

7. Chiarioni G, Whitehead WE, Bassotti G. Randomized controlled trial of biofeedback. Clin Gastroenterol Hepatol 2007;5:1119.

8. Rao SS. Dyssynergic defecation and biofeedback therapy. Gastroenterol Clin North Am 2008;37:569-586,viii.

9. Chiarioni G, Salandini L, Whitehead WE. Biofeedback benefits only patients with outlet dysfunction, not patients with isolated slow transit constipation. Gastroenterology 2005;129:86-97.

10. Park DH, Myung SJ, Yoon IJ, et al. Clinical factors associated with response to biofeedback therapy for patients with chronic constipation. Korean J Gastroenterol 2003;42:289-296.

11. Chiarioni G, Whitehead WE, Pezza V, Morelli A, Bassotti G. 
Biofeedback is superior to laxatives for normal transit constipation due to pelvic floor dyssynergia. Gastroenterology 2006;130:657-664.

12. Gladman MA, Lunniss PJ, Scott SM, Swash M. Rectal hyposensitivity. Am J Gastroenterol 2006;101:1140-1151.

13. Chang HS, Myung SJ, Yang SK, et al. Effect of electrical stim- ulation in constipated patients with impaired rectal sensation. Int $\mathrm{J}$ Colorectal Dis 2003; 18:433-438.

14. Chang HS, Myung SJ, Yang SK, et al. Functional constipation with impaired rectal sensation improved by electrical stimulation therapy: report of a case. Dis Colon Rectum 2004;47:933-936. 